\title{
Learning in depth with the bespoke rubric-supported online poster presentation
}

\section{Alireza Lajevardipour, Andrew Wood}

Alireza Lajevardipour, Andrew Wood, "Learning in depth with the bespoke rubric-supported online poster presentation," Proc. SPIE 10452, 14th Conference on Education and Training in Optics and Photonics: ETOP 2017, 104521R (16 August 2017); doi: 10.1117/12.2266475

SPIE Event: 14th Conference on Education and Training in Optics and Photonics, ETOP 2017, 2017, Hangzhou, China 


\title{
Learning in depth with the bespoke rubric-supported online poster presentation
}

\author{
Alireza Lajevardipour ${ }^{\dagger a}$, Andrew Wood ${ }^{\ddagger a}$ \\ ${ }^{\text {a }}$ School of Health Sciences, Swinburne University of Technology, Melbourne, Australia \\ $\dagger$ alajevardipour@swin.edu.au; $\$$ awood@swin.edu.au
}

\begin{abstract}
In our course of Biomedical Imaging, we introduced a research project as an assignment that included an online poster presentation. To assess the assignment, an adjusted criteria sheet was created, where it facilitated providing students with an effective feedback linked to particular criteria. Students are expected to produce a scientific poster to present the result of their investigation and upload it to an online discussion board. In addition, they are required to read their colleagues' works and provide peer-feedback by asking quality questions about principles and results, also on-line. Subtle distribution of marks in the rubric balances focus between preparing poster and providing peer-feedbacks.
\end{abstract}

Keyword list: bespoke rubric, online poster presentation, Biomedical Imaging course, peer-feedback, assessment

\section{INTRODUCTION}

The authors have been involved in teaching a 1-semester course named "Biomedical Imaging". The course was designed for undergraduate students in health science and biotechnology disciplines. It aimed to provide a solid introduction to fundamental concepts of major imaging modalities currently used in clinical and biomedical research settings and relate them to cutting edge technologies and their possible applications to medicine.

In recent years along with advances in photonics, the number of available biomedical imaging techniques have burgeoned. There are variety of imaging methods and techniques that are suitable for particular situations. It makes it impossible to cover all methods and related cases in a single semester course. Therefore, encouraging students to engage in self-discovery of related topics and share them with their peers is an efficient alternative way. To address this issue, a research project was added to the course as a Teaching and Learning Activity (TLA).

Constructive alignment ${ }^{1}$ requires that all TLAs included assessment task support Intended Learning Outcomes (ILOs) of the course. In other word, assessment tasks are treated as a learning activity and should be merged into curriculum. As Ramsden ${ }^{2}$ mentioned, students will learn what they think they will be assessed on. Thus inserting the research project to the course will be effective, providing its associated assessment can be fully merged into the course curriculum.

\section{POSTER ASSIGNMENT}

In this research project activity, students are required to select three preferred research subjects from a range of research topics and register them in the early weeks of the semester. They are promptly assigned to a topic from their preferences. Then they have enough time in the rest of semester to think about the topic and find a method to study and investigate their chosen subject individually. The number of topics in the list is related to the number of students in the class where the maximum of three students work on a common topic independently. The list of topics covers different areas in the biomedical imaging field, focusing on advanced microscopy methods. The following items display a typical list of topics:

1. The use of quantum dot imaging in studying cell function

2. The use of super-resolution microscopy to study cell function

3. Use of FRET to study protein-protein interactions in cells

14th Conference on Education and Training in Optics and Photonics: ETOP 2017, edited by Xu Liu,

Xi-Cheng Zhang, Proc. of SPIE Vol. 10452, 104521R - (c) 2017 ICO, IEEE, OSA, SPIE

CCC code: $0277-786 X / 17 / \$ 18 \cdot$ doi: $10.1117 / 12.2266475$

Proc. of SPIE Vol. 10452 104521R-1 
4. The use of fluctuation techniques to study the motions of molecules

5. Fluorescence optical imaging of live animals

6. The imaging of temperature changes within living organs

7. Fluorescence imaging of dynamic structures

8. Label-free imaging using intrinsic photonic properties

9. Fluorescent biosensors for networks and pathways

10. Single molecule imaging at the plasma membrane

11. Microwave imaging for breast cancer detection

12. Medical applications of terahertz radiation, imaging in particular

13. Principles and biomedical applications of Atomic Force Microscopy

14. Carbon nanotubes - possible applications in biomedical imaging

15. Imaging the gut using a radio-transmitting device ('Pill-cam')

16. Interfacing neurons with silicon microcircuits: checking structure

17. A multi-technique biosensor device to detect and treat a disease

18. Nano-diamonds as optical probes in bio-imaging

Students are expected to produce a scientific poster to present the result of their investigation and upload it to an online discussion board a month before the end of semester. Therefore, there is time for other students to read their colleagues' work and provide peer-feedback by asking quality questions about principles and results, also on-line. As a supplementary activity, they are also expected to provide peer-feedback for at least four other posters in two topics and respond to the questions asked of them.

Poster should highlight the main points on assigned topic and be limited to one A4 page. Its style must comply with logical progression from introduction to conclusion. All posters must be posted on the online Discussion Board facility (Blackboard) through a plagiarism detection system called Turnitin.

Other part of assignment is providing peer-feedback and responses. Students must also read four other posters from their own assigned topic and one other topic. They are assigned posters that must look at and ask questions on. Each student can expect around four questions, which should be answered as precisely as possible. The 100 marks for the assignment are apportioned based on the rubric. Students who assigned the same topic are encouraged to reply questions even related to work of other students. It promotes a discussion among students.

Students' performance is assessed by two different assessors and in the case of big difference between assessors the third assessor will be employed. All assessment is based on a rubric. Feedback is provided to students from the assessors via the Blackboard.

\section{A TYPICAL DISCUSSION THREAD}

The complementary part of the assignment is attending online poster presentation by providing feedbacks for peers and responding to the asked questions. This part increases discussions among students. Some typical questions and answers are as the following:

Topic of the use of super-resolution microscopy to study cell function

"Hi Joe Doe

Well done! What are the limitations of the Super Resolution Microscopy when it comes to study biological Structures? Thanks."

"Hi Jane

Thanks! 
The limitation of SRM has improved both lateral and axial resolutions, achieving 50-70nm resolution in all three dimensions. Hence, the molecular resolution is roughly about $1-5 \mathrm{~nm}$. Living cells potentially move faster than super resolution microscopes can capture images. As the latest new super resolution technologies are based on illumination, nonlinear fluorophore responses and the localisation of single molecules enable to investigate the structure and function of biological cells."

Topic of Nano-diamonds as optical probes in bio-imaging

"Hi Jeff,

Based on your research, I understand that nitrogen vacancy centre in the nanodiamond has the function of fluorescence and it is important part for its use. My question is: How is the NV centre (a flaw in nanodaiamond) made? Do we just need to find the nanodiamond particles that have a NV centre from naturally formed nanodiamonds or is it possible to have man-made NV centres? If so, how is it made?

Regards, Rob"

"Hello Rob,

Thank you for your question./

There are two possible ways to create the nitrogen vacancy centres. One way is through the use of Chemical Vapour

Deposition, which is a chemical process in which deposition of gaseous reactants on to a substrate occurs, in this case the nitrogen donor molecules is immobile from the deposition where as the vacancies in the nanodiamond are highly mobile and can therefore easily undergo diffusion of the lattice. Another way that nitrogen vacancy centre is produced is through the growth of the nitrogen vacancy into the centre as a unit as their creation pertaining to process occur on the growth surface.

I hope that answers your question

Cheers, Jeff"

These typical questions and answers demonstrate how peer-discussion works, where these interactions can ultimately deepen learning by supporting ILOs.

\section{THE RUBRIC}

Criterion-referenced assessment can be implemented to avoid comparing students' works and reference to the achievement of others in the class. This method of assessment is the process of evaluating product of students against a set of pre-specified criteria ${ }^{3}$. This criteria sheet is known as grading rubrics and is the central part of criterion-referenced assessment.

The rubric facilitates providing students with an effective feedback linked to particular criteria. It reduces effort to deliver feedback and enhances its quality. Although rubric is time-consuming to construct it is time-saving to use for both students and instructors. Table 1 demonstrates the bespoke rubric related to the research project assignment. It is comprised of five levels of achievement that are generally used at Swinburne University of Technology includes High Distinction, Distinction, Credit, Pass and Fail.

\section{DISCUSSION}

The introduced project activity exposes learners to a new complex and challenging learning situation to study about advanced microscopy techniques. Where it deepens learning by engaging students to go through fundamental concepts of imaging method and related physics. This TLA is an engaging item in the course with introducing contemporary topics of Nano and Bio photonics applications in Biomedical Imaging. Moreover, it exposes students to a complex situation that need to be handled by learning new skills like scientific writing and preparing scientific posters. One can call this process "learning through production" or collaborative learning ${ }^{4}$. 
The project is a high-level task with well-defined expectations set out in a complementary rubric. The bespoke rubric includes statements of standard descriptors and weighting for each criterion to encompass the intents of criterionreferenced assessment. It allows students to evaluate their own work before submission and lets instructors to mark posters without comparison.

The marks for the assignment divided into two major parts, one preparing poster itself and second, providing and responding to feedbacks. This way of distributing marks emphasises that reacting to poster presentations is unneglectable part of assignment and students need to consider this part to receive desirable mark.

The rubric enhances summative assessment by enabling detailed grading based on a set of pre-specified criteria. In addition, it promotes compatibility of judgment between two assessors and reduces the frequency of having to refer to a third assessor. Moreover, the rubric can facilitate providing feedback by linking to relevant criteria that will consequently create formative assessment. Further, it aligns the project activity with Intended Learning Outcomes (ILOs) of the course. Using online learning delivery and management system for poster presentation and discussion changes the delivery of the course from merely face-to-face mode to a blended mode where it boosts the efficiency of presentation in information interchange among students to provide peer-feedback and enhances the quality of grading based on documented action and reaction in presentation. Ultimately, the provided feedbacks empower students to regulate aspects of their approach to research the topic within a model of self-regulated learning ${ }^{5}$.

\section{CONCLUSION}

From these advantages, we can reach the conclusion that the project activity along with its adjusted rubric can deepen learning in the Biomedical Imaging course, where, introduced rubric and requirement to react on feedbacks lead students to be self-regulated learners.

\section{REFERENCES}

[1] Biggs, J. B. and Tang, C. S., [Teaching for quality learning at university: What the student does], McGraw-Hill, Maidenhead, (2011).

[2] Ramsden, P., [Learning to teach in higher education], Routledge, London, (2003).

[3] Taylor, C., "Assessment for Measurement or Standards: The Peril and Promise of Large-Scale Assessment Reform," American Educational Research Journal 31(2), 231-62 (1994).

[4] Laurillard, D., [Teaching as a design science: Building pedagogical patterns for learning and technology], Routledge, New York, (2012).

[5] Sadler, D. R., "Formative assessment and the design of instructional systems," Instr. Sci. 18(2), 119-44 (1989). 


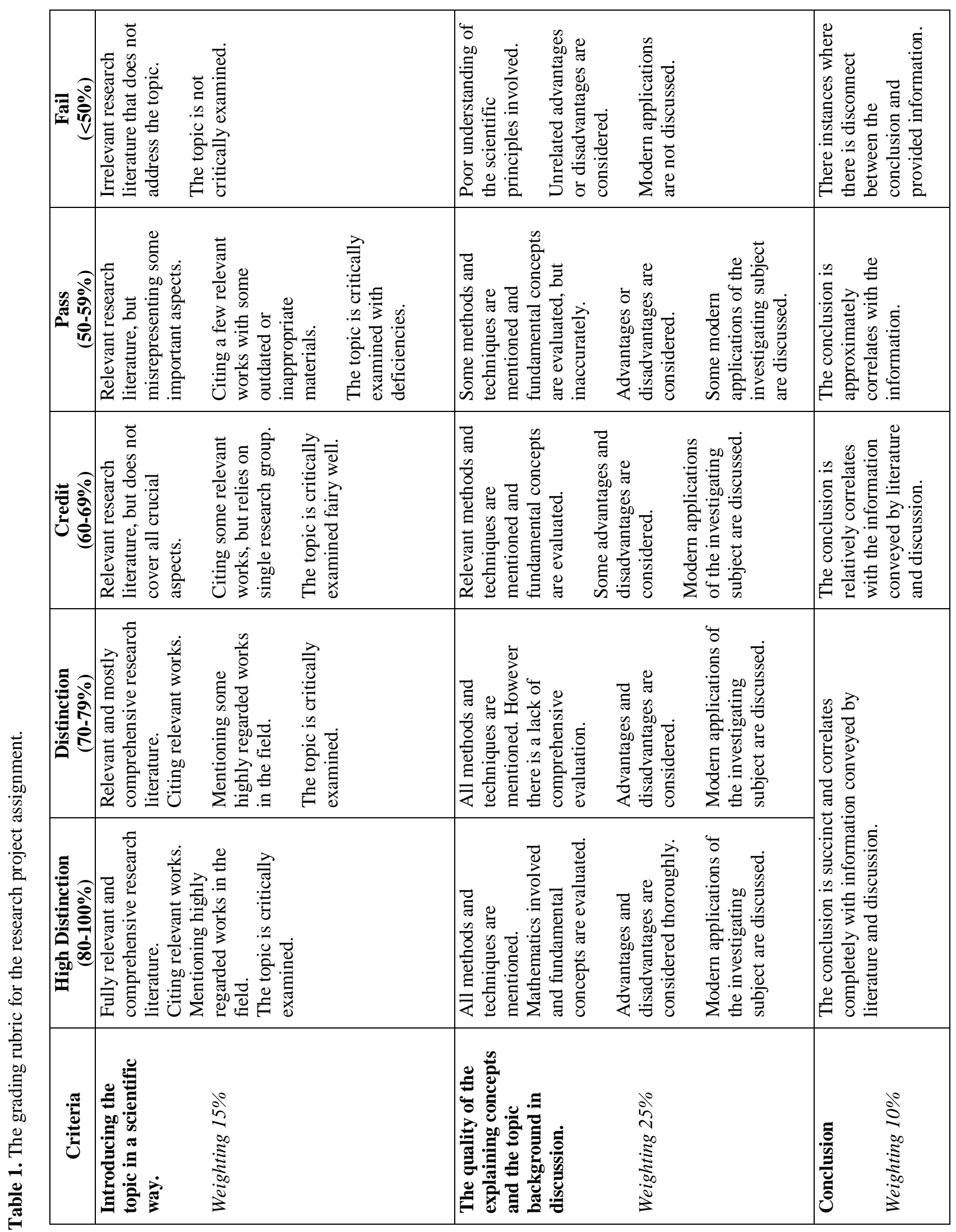




\begin{tabular}{|c|c|}
\hline 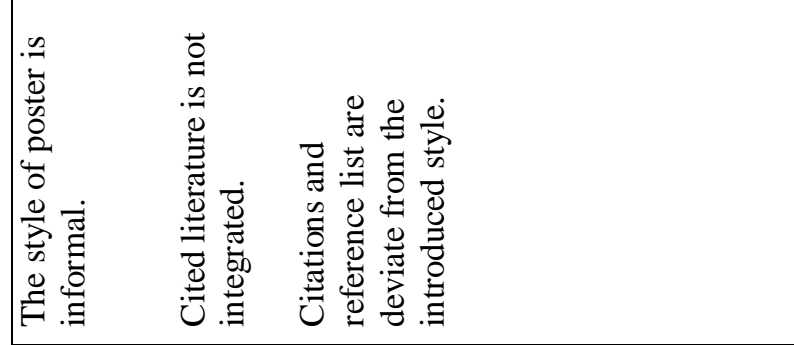 & 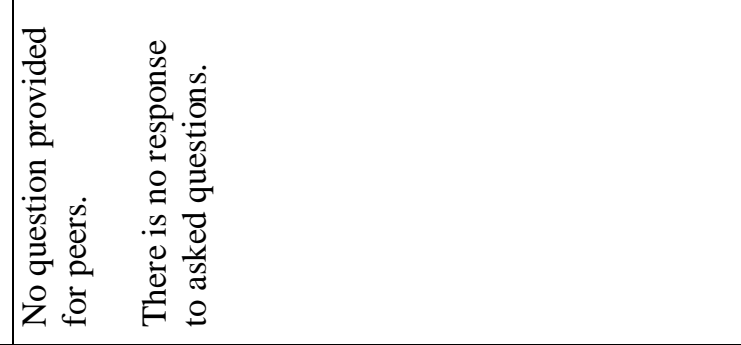 \\
\hline 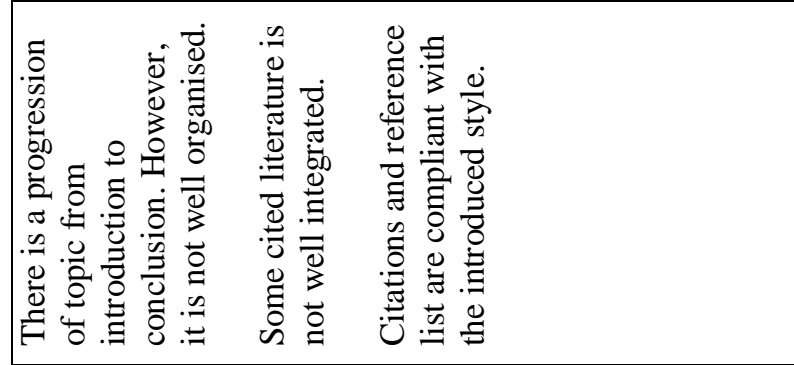 & 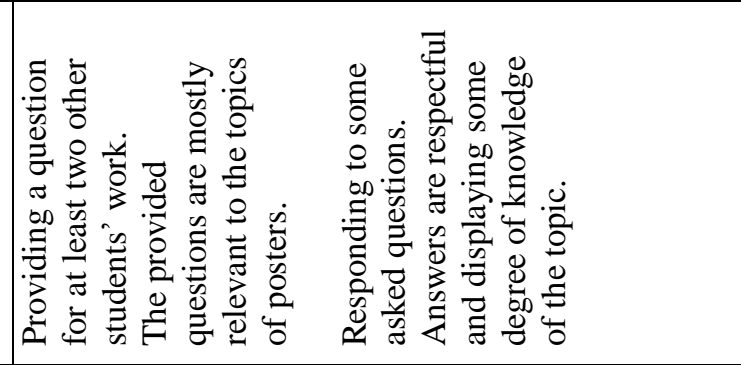 \\
\hline 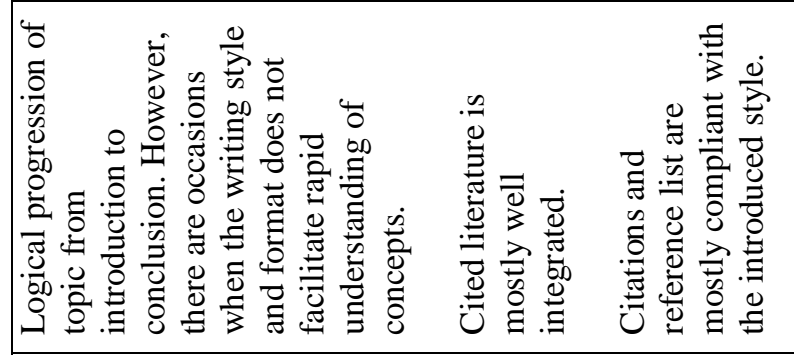 & 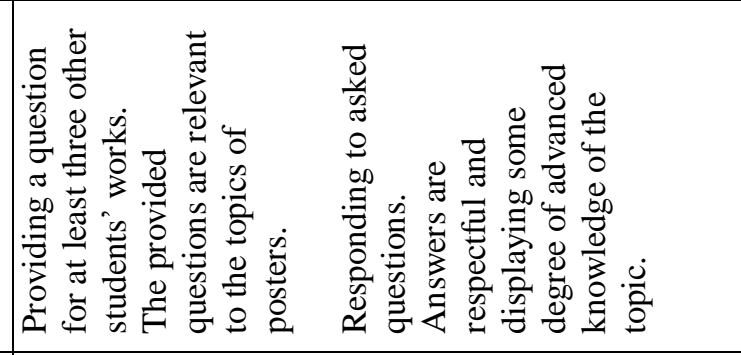 \\
\hline 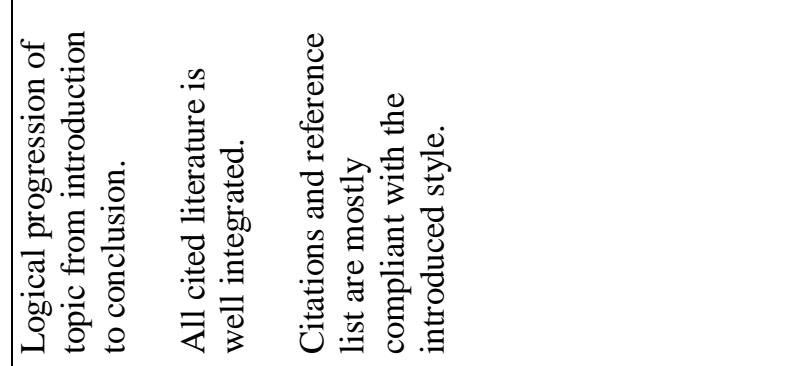 & 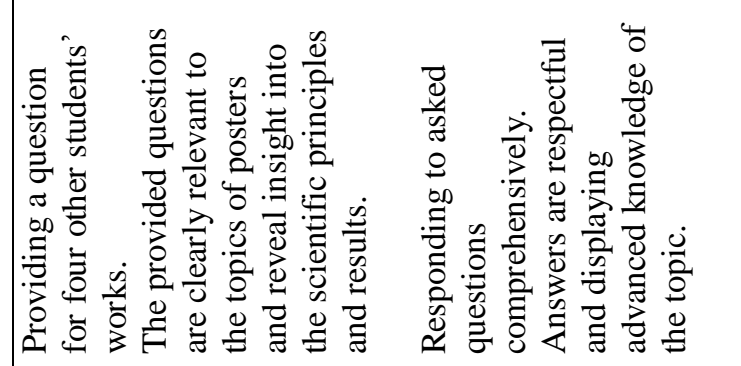 \\
\hline 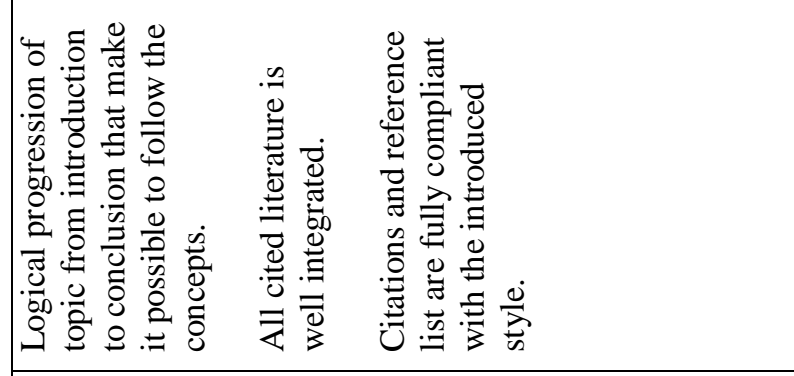 & 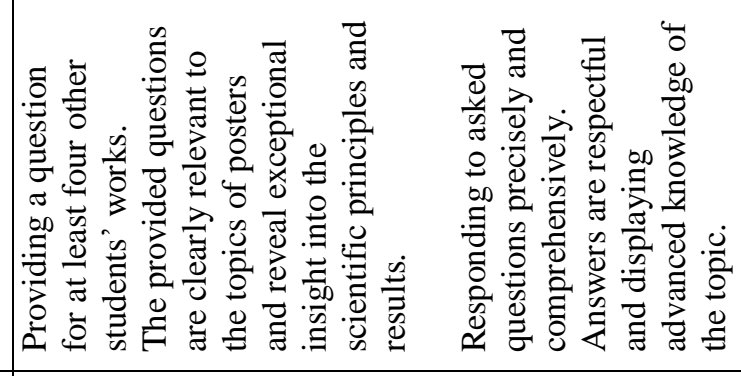 \\
\hline 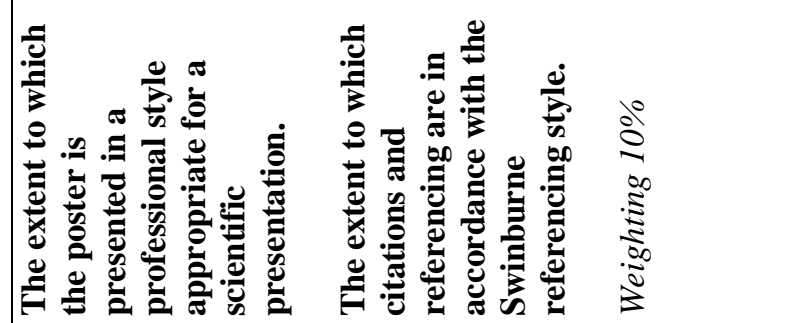 & 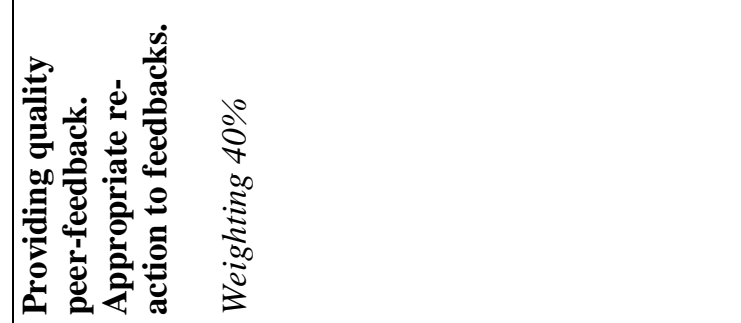 \\
\hline
\end{tabular}

